\title{
An exploratory study on the efficacy and safety of a BCAA preparation used in combination with cardiac rehabilitation for patients with chronic heart failure
}

\author{
Munenori Takata ${ }^{1,2^{*}}$, Eisuke Amiya ${ }^{1 *}$ (1), Masafumi Watanabe ${ }^{1}$, Yumiko Hosoya ${ }^{1}$, Atsuko Nakayama ${ }^{1}$, \\ Takayuki Fujiwara', Masanobu Taya ${ }^{1,4}$, Gaku Oguri ${ }^{1,2}$, Kanako Hyodo ${ }^{3}$, Naoko Takayama ${ }^{3}$, Nami Takano ${ }^{1}$, \\ Tomoe Mashiko ${ }^{5}$, Yukari Uemura ${ }^{2}$ and Issei Komuro ${ }^{1}$
}

\begin{abstract}
Background: Sarcopenia is generally complicated with patients with chronic heart failure (CHF) and its presence negatively affects the course of heart failure, however effective nutritional intervention had not been elucidated yet. The primary objective of this study is to explore whether the addition of a branched-chain amino acid (BCAA) preparation for cardiac rehabilitation (CR) of patients with CHF further improves cardiopulmonary functions, skeletal muscle functions, and metabolism in comparison with conventional CR.

Methods: This is a randomized, parallel-group comparative study. The elderly patients that were participated in CR and complicated with left ventricular systolic or diastolic dysfunction are randomized into two groups, CR + BCAA and CR. 20 weeks later, the second randomization is performed, which divide subjects into two groups with and without BCAA intervention without CR. Primary outcome measure is the rate of change of the anaerobic threshold workload from baseline to post-intervention. Secondary outcome include parameters of exercise capacity, cardiac function and psychological status.

Discussion: In the current study the effect of a promising new intervention, BCAA, will be assessed to determine whether its addition to CR improve exercise capacity in patients with heart failure, who are generally complicated with sarcopenia.
\end{abstract}

Trial registration: This clinical trial was registered with the University Hospital Medical Information Network—Clinical Trials Registry (UMIN-CTR; JPRN-UMIN R000022440).

\section{Background}

Shortness of breath and labored breathing occur during daily activities in patients with chronic heart failure (CHF). These symptoms are caused by decreased systolic and diastolic cardiac function. Decreased exercise tolerance because of heart failure is also likely to be complicated with skeletal muscle weakness [1]. Indeed, the exercise tolerance of patients with $\mathrm{CHF}$ is known to be strongly related to skeletal muscle strength and muscle

\footnotetext{
*Correspondence: takatam-tky@umin.ac.jp; amiyae-tky@umin.ac.jp ${ }^{1}$ Department of Cardiovascular Medicine, Graduate School of Medicine, The University of Tokyo, 7-3-1 Hongo, Bunkyo-ku, Tokyo 113-8655, Japan

Full list of author information is available at the end of the article
}

mass [2]. A decrease in skeletal muscle strength and muscle mass (sarcopenia) is an important factor that largely affects the subjective symptoms of patients with CHF. Furthermore, sarcopenia is generally complicated with several abnormalities such as metabolic disorders and decreased vascular function [3]. Sarcopenia is often induced by physical deconditioning because of rest during CHF treatment, and it can lead to a condition known as cachexia if concurrent metabolic disorders exist. Approximately $20 \%$ CHF patients are believed to suffer from sarcopenia, whereas $10 \%$ suffer from cachexia [4], and both are generally observed in elderly patients with cardiovascular disease [5]. Japan is experiencing an 
increasingly aging population; thus, CHF with coexisting sarcopenia and cachexia have become a big burden.

From the fact that $10 \%-50 \%$ of CHF patients who undergo a single pharmacotherapy intervention are repeatedly admitted for heart failure within a period of 36 months, it is evident that the presently available CHF treatments are quite insufficient. With a focus on sarcopenia and cachexia, cardiac rehabilitation is another comprehensive approach that improves cardiopulmonary functions, leading to the suppression heart failure events. Furthermore, in addition to an increase in exercise tolerance, cardiac rehabilitation has been shown to improve left ventricular function, endothelial function, and sympathetic activity, which may have favorable effects on the course of heart failure [6-10].

Branched-chain amino acid (BCAA) preparations have been available for purchase in the market since 1996 with the aim of correcting amino acid imbalance in the blood in patients with decompensated cirrhosis and improving hypoalbuminemia. BCAAs play an important role in the formation of skeletal muscles because they account for approximately $35 \%$ of the essential amino acids that form these skeletal muscles [11]. Indeed, BCAA preparations have been reported to promote postoperative wound healing and recovery from muscle fatigue after exercise and enhance muscle strength [12]. Its easily absorbable trait may also fit the treatment of heart failure, because patients with CHF often decreased digestive abilities because of cachexia. Furthermore, reports from animal experiments have revealed that BCAA had some positive effects on heart failure through the improvement on the function of the skeletal muscle mitochondria [13, 14]. Therefore, incorporation of BCAA in cardiac rehabilitation is expected to have additional effect on the improvement of cardiopulmonary and skeletal muscle functions. In this study, we hypothesized that the concurrent use of a BCAA preparation during cardiac rehabilitation has synergistic effects on the clinical course of $\mathrm{CHF}$ as well as improvement of skeletal muscle function, and metabolism. In addition, we would also like to investigate whether continued administration of the BCAA preparation after cardiac rehabilitation preserves cardiopulmonary function and skeletal muscles.

\section{Aim}

The primary objective of this study is to explore whether the addition of a BCAA preparation for cardiac rehabilitation of patients with CHF further improves cardiopulmonary functions, skeletal muscle functions, and metabolism in comparison with conventional cardiac rehabilitation. In addition, we will also explore whether continued administration of a BCAA preparation after cardiac rehabilitation can preserve cardiopulmonary and skeletal muscles functions.

\section{Methods/Design \\ Patient enrollment}

Patients with CHF was evaluated in the University of Tokyo Hospital and recruited if they fulfilled the following criteria: (1) Participation in a monitored cardiac rehabilitation program for CHF, (2) age $\geq 65$ years, (3) experiencing left ventricular systolic dysfunction [ejection fraction (EF) $<40 \%$ on echocardiography] or diastolic dysfunction (EF $>40 \%$ and $\left.\mathrm{E} / \mathrm{e}^{\prime}>15\right)$.

\section{Exclusion criteria}

A patient who meets any of the following criteria will not be included in the study: (1) presence of a congenital BCAA metabolism disorder, (2) presence of contraindications to cardiac rehabilitation, (3) pregnant or breastfeeding patient, (4) presence of other conditions, which make the patient unsuitable for the study according to the judgment of the principal investigator (PI) or coinvestigator (Co-I).

\section{Study methods}

This is a randomized, active-controlled, parallel-group comparative study. Study outline is presented in Fig. 1. The study consists of three periods-pre-observation, intervention with concurrent cardiac rehabilitation, and BCAA single intervention. In pre-observation period (Day 1 to Week 4), the consent of patients undergoing cardiac rehabilitation at the hospital for CHF will be obtained, and their eligibility will be determined by blood tests and imaging tests (echocardiogram). In the period of intervention with concurrent cardiac rehabilitation (for 18-22 weeks), patients who are confirmed eligible for study enrollment will be randomized into either group A $(\mathrm{CR}+\mathrm{BCAA})$ or group B (CR). BCAA is orally supplemented with one pack of BCAAs granules (Aminovact Combination Granules ', Nichi-Iko Pharmaceutical Co, Toyama, Japan) which includes $1144 \mathrm{mg}$ of L-valine, $1904 \mathrm{mg}$ of L-leucine and $952 \mathrm{mg}$ of L-isoleucine, two times a day. Cardiopulmonary exercise test (CPX) will be performed on each subject in both groups before intervention with the BCAA preparation as well as at Week 14 and Week 20 of the intervention with cardiac rehabilitation. In the BCAA preparation single intervention period (20-24 weeks), re-randomization, i.e., randomization 2 , will be performed after the cardiac rehabilitation program has ended. Subjects in group A will be assigned to Group A-1 or A-2, whereas those in Group B will be assigned to Group B-1 or B-2. As described in Fig. 1, subjects assigned to Group A-1 will continue the BCAA preparation. Similarly, subjects in Group B-1 will start taking the BCAA preparation. The BCAA preparation will be discontinued for subjects assigned to Group A-2, and subjects in Group B-2 will not undergo intervention with the BCAA preparation throughout the study period. 


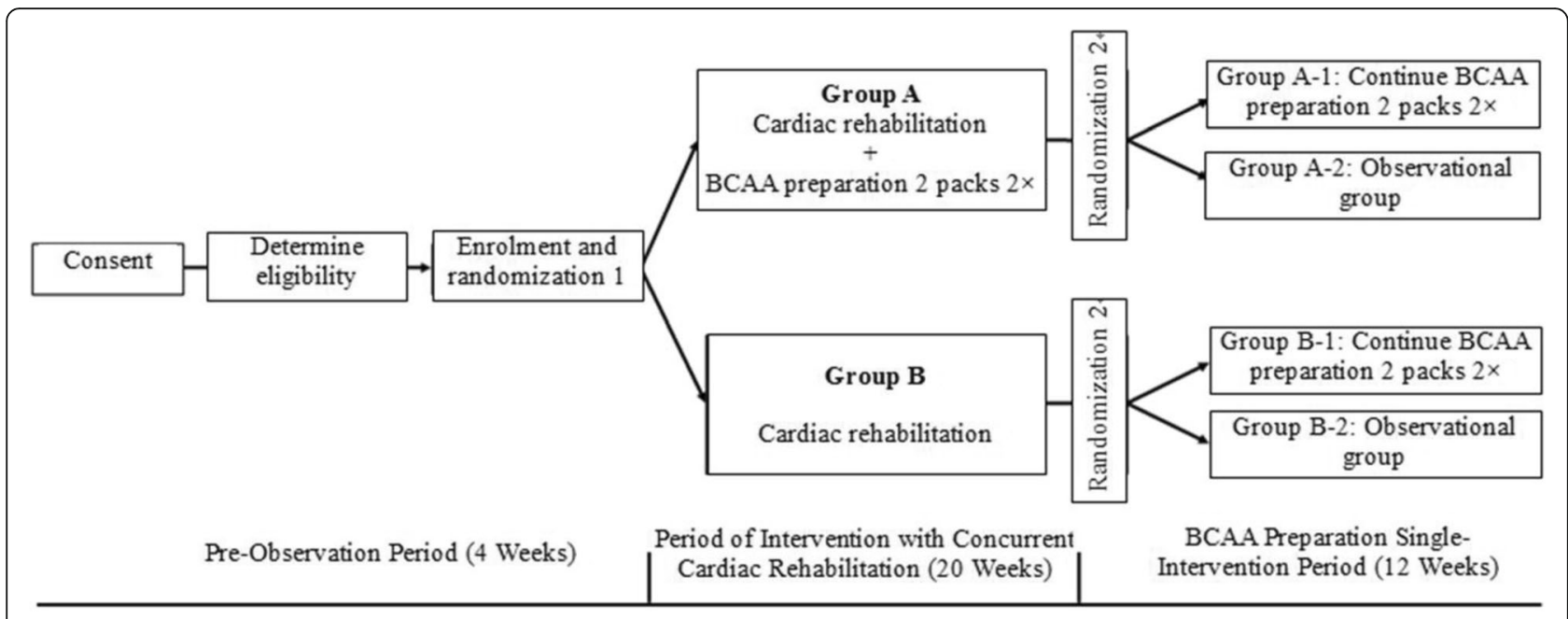

Fig. 1 Schematic of study design

This clinical study is in accordance with International Conference on Harmonization of technical requirements for registration of pharmaceuticals for human use (ICHGCP). Furthermore, the clinical study is conducted with strict observance of ethical guidelines and the latest Declaration of Helsinki. This clinical trial was registered with the University Hospital Medical Information Network-Clinical Trials Registry (UMIN-CTR; JPRNUMIN R000022440). Also, the protocol was approved by the institutional review board of Tokyo University Hospital.

\section{Enrollment and assignment}

PI or Co-I will obtain the written consent of subjects. The enrollment of subjects who have given their consent and the data of all subjects will be entered into the electronic data capture (EDC) system (Viedoc, PCG Solutions, Uppsala, Sweden). For randomization 1, the subjects will be stratified into two groups (groups A and $\mathrm{B}$ as mentioned earlier) according to (1) age ( $>70$ or $\leq 70$ years) and (2) anaerobic threshold (AT) workload ( $\geq 30$ watts or $<30$ watts) in the first CPX performed at the time of consent-taking. Randomization 2 will be performed after the intervention period with concurrent cardiac rehabilitation has ended. The stratification factors are (1) the age of the subject ( $>70$ or $\leq 70$ years) and (2) the rate of change in the AT workload in CPX after intervention with concurrent cardiac rehabilitation has ended ( $\geq 25 \%$ or $<25 \%$ for group A and $\geq 15 \%$ or $<15 \%$ for group B).

\section{Outcome measures}

Primary outcome measure is the rate of change of the AT workload from baseline to post-intervention with concurrent cardiac rehabilitation. Secondary outcome measures include the following: (1) The rate of change from baseline which is performed in the pre-observation period or reference day [Peak $\mathrm{VO}_{2}$, left ventricular EF in the echocardiogram, total points of the Hospital Anxiety and Depression Scale (HADS), muscle strength (grip strength and lower-limb extension power), absolute values of interleukin-6 (IL-6), and tumor necrosis factor (TNF)- $\alpha$ ], Time taken for $50 \%$ subjects to achieve a $15 \%$ improvement in AT workload, which is defined as an event. (2) The rate of change from post-intervention period with cardiac rehabilitation (20 weeks later) to post-single intervention with the BCAA preparation (32 weeks later) [Peak $\mathrm{VO}_{2}$, AT workload, total points of HADS, muscle strength (grip strength and lower-limb extension power))], (3) The frequency of serious adverse events (SAEs) throughout the study period.

\section{Observations and examinations}

After enrollment, PI or Co-I collect all data prospectively whenever feasible. Data include the following: (1) the patient's background: gender, date of birth, height, body weight, complications, past medical history, etiology of heart failure, coronary risk factors (hypertension, dyslipidemia, diabetes, hyperuricemia, and age > 45 years), and history of present illnesses; (2) compliance to IP administration (records of the day on which the BCAA preparation was first prescribed and the day of the last prescription) and the number of visits to the hospital for cardiac rehabilitation (per week); (3) concomitant medications; (4) subjective symptoms (Borg scale during cardiac rehabilitation) and objective findings; (5) adverse events $(\mathrm{AE})^{\mathrm{a}}$ : details of $\mathrm{AE}$, date and time of $\mathrm{AE}$ onset and resolution, severity, treatments, outcome, assessment of severity, and causal relationship with IP will be entered into the medical records and EDC. Follow-up investigations will 
be performed, if necessary. The levels of severity are defined as follows: (1) mild: treatment is not required and IP administration can continue, (2) moderate: IP administration can continue with some treatment, and (3) severe: IP administration will be or should be discontinued.

Characteristic patient data includes following parameters (Table 1): (1) Vital signs: Blood pressure before the start of cardiac rehabilitation (before warm-up exercise), pulse rate (in sitting position), as well as blood pressure and pulse rate at the time of outpatient visits (in sitting position); (2) $\mathrm{CPX}^{\mathrm{b}}$ : AT workload, $\mathrm{VO}_{2} \mathrm{AT}$, peak $\mathrm{VO}_{2}$, and $\Delta \mathrm{VO}_{2} / \Delta$ work rate (WR); (3) Complete blood count $(\mathrm{CBC})$, biochemistry tests and blood plasma tests ${ }^{\mathrm{c}}$ : White blood cell, red blood cell, hemoglobin, hematocrit, platelet, total protein, albumin, cholinesterase, aspartate aminotransferase, alanine aminotransaminase, gammaglutamyl transpeptidase, blood urea nitrogen, creatinine, C-reactive protein, and brain natriuretic peptide; (4) Cytokine blood tests ${ }^{\mathrm{d}}$ : TNF- $\alpha$ and IL-6; (5) Echocardiography ${ }^{\mathrm{e}}$ : left ventricular diastolic diameter (M mode), left ventricular EF (M mode), E/A, E/e', and left atrial diameter; (6) Emotional outcome measure ${ }^{f}$ : Total points of HADS; (7) Assessment of muscle strength ${ }^{\mathrm{g}}$ : Grip strength and lower-limb extension power; (8) Events of hospitalization because of heart failure during the study period.

\section{Statistical considerations}

The sample size was calculated according to the estimation as follows. We hypothesized that following 6 months of cardiac rehabilitation, there will be an AT workload improvement of $25 \%$ (SD: $10 \%$ ). With the addition of BCAA, we hypothesized that there will be an AT workload improvement of $25 \%$ (SD: $10 \%)$. Setting the twosided significance level at $5 \%$ and power at $90 \%$, the required sample size was 43 cases. Assuming a 5\% rate of subjects leaving the study, we chose 48 cases as the target number.

According to the study results, differences (\%) in primary outcome between each outcome measurement

Table 1 Study schedule

\begin{tabular}{|c|c|c|c|c|c|c|c|c|}
\hline \multicolumn{2}{|c|}{ Item } & \multirow{2}{*}{$\begin{array}{c}\begin{array}{c}\text { Previous } \\
\text { observation } \\
\text { period }\end{array} \\
1 \text { day to } 4 \\
\text { weeks } \\
\text { earlier }\end{array}$} & \multirow{2}{*}{$\begin{array}{c}\begin{array}{c}\text { Date of } \\
\text { treatment } \\
\text { initiation }\end{array} \\
\text { Day 0 }\end{array}$} & \multicolumn{4}{|c|}{ Duration of concurrent cardiac rehabilitation } & \multirow{2}{*}{$\begin{array}{c}\text { Duration of } \\
\text { BCAA only } \\
\begin{array}{c}\text { Termination of } \\
\text { treatment at } 32 \pm 4 \\
\text { weeks }\end{array}\end{array}$} \\
\hline & Period & & & $\begin{array}{c}\text { After } \\
\text { treatme } \\
\text { nt of } 4 \\
\pm 1 \\
\text { week(s) }\end{array}$ & $\begin{array}{c}\text { After } \\
\text { treatment of } \\
8 \pm 2 \text { weeks }\end{array}$ & $\begin{array}{c}\text { After } \\
\text { treatment of } \\
14 \pm 2 \\
\text { weeks }\end{array}$ & $\begin{array}{c}\text { After } \\
\text { treatment of } \\
20 \pm 2 \\
\text { weeks }\end{array}$ & \\
\hline \multicolumn{2}{|r|}{ Checkup } & Visit 1 & Visit 2 & Visit 3 & Visit 4 & Visit 5 & Visit 6 & Visit 7 \\
\hline \multicolumn{2}{|c|}{ Patient consent } & O & & & & & & \\
\hline \multicolumn{2}{|c|}{$\begin{array}{c}\text { Confirmation of } \\
\text { patient clinical } \\
\text { history }\end{array}$} & $\bigcirc$ & & & & & & \\
\hline \multicolumn{2}{|c|}{ Randomization } & & $\bigcirc$ & & & & ○ & \\
\hline \multicolumn{2}{|c|}{$\begin{array}{l}\text { Administration } \\
\text { of BCAA }\end{array}$} & & & & & & & \\
\hline \multicolumn{2}{|c|}{$\begin{array}{l}\text { Physical } \\
\text { examination }\end{array}$} & $\bigcirc$ & 0 & 0 & 0 & ○ & ○ & ○ \\
\hline \multicolumn{2}{|c|}{ Adverse events ${ }^{\mathrm{a}}$} & & & & & & & \\
\hline \multicolumn{2}{|r|}{ Vital signs } & O & O & 0 & O & 0 & O & O \\
\hline \multicolumn{2}{|r|}{$\mathrm{CPX}^{\mathrm{b}}$} & & $\bigcirc$ & & & ○ & ○ & 0 \\
\hline \multirow{2}{*}{ 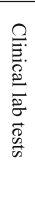 } & $\begin{array}{l}\text { CBC } \cdot \\
\text { chemistry } \\
\text { panel } \cdot \\
\text { plasma }^{\text {c }}\end{array}$ & $\bigcirc$ & & & O & O & ○ & O \\
\hline & $\begin{array}{l}\text { cytokine } \\
\text { blood tests }^{\mathrm{d}}\end{array}$ & O & & & & & ○ & \% \\
\hline \multicolumn{2}{|c|}{$\begin{array}{c}\text { Echocardiograp } \\
\text { hy }^{\mathrm{e}}\end{array}$} & O & & & & & 0 & \\
\hline \multicolumn{2}{|c|}{$\begin{array}{c}\text { Psychological } \\
\text { factors assessed }\end{array}$} & O & & & & & 0 & 0 \\
\hline \multicolumn{2}{|c|}{$\begin{array}{c}\text { Muscle volume } \\
\text { assessed }^{\mathrm{g}}\end{array}$} & 0 & & & & & 0 & 0 \\
\hline \multicolumn{2}{|c|}{$\begin{array}{l}\text { Concurrent drug } \\
\text { administered }\end{array}$} & & & & & & & \\
\hline
\end{tabular}

- Indicates items that occur before administration of BCAA

- Indicates items that occur after administration of BCAA

※ Indicates items that occur after suspension of the study 
between $\mathrm{A}$ and $\mathrm{B}$ groups will be evaluated using the $\mathrm{t}$ test. With respect to differences in outcome measurement (\%) in secondary endpoint between groups A and $B$, the t-test will be used to analyze statistical significance. Similarly, the difference between B-1 and B-2 sub-groups will be analyzed using the t-test. Also,, after confirming the assumption that there are no major differences among the treatment groups (no interaction), the two-way ANOVA will be used to test the treatment effect for addition of a BCAA, and maintenance effect for continuing administration of a BCAA by including group $\mathrm{A} / \mathrm{B}$ and $+1 /+2$ as factors. A box-plot will be used to visualize primary outcome and secondary outcomes between the 4 groups $(\mathrm{A}+1, \mathrm{~A}+2, \mathrm{~B}+1, \mathrm{~B}+2)$. Finally, defining an AT workload improvement of $15 \%$ as an event, a Kaplan-Meier curve will be plotted to compare the curves between groups $\mathrm{A}$ and $\mathrm{B}$, and estimate the occurrence of an event rate of $50 \%$ of each group.

\section{Discussion}

Cardiac rehabilitation includes nutritional interventions in addition to exercise training [15]. The main aim of nutritional intervention seems to be an assist of effect derived from exercise training interventions; however, the nutritional modification might itself have favorable effects on the skeletal muscle or cardiopulmonary capacity leading to the improvement of exercise capacity in patients with heart failure [16]. The study focused on the elderly patients with heart failure, who are potentially complicated with sarcopenia. However, we did not limit the study patients by excluding patients without subjectively confirmed sarcopenia because we would investigate the effectiveness of more general and easy use of BCAA agents for cardiovascular patients.

One unique point in this study is that there are two terms of nutritional interventions, one with exercise training and one without it. The results help distinguishing the additional effect on exercise training or original effect derived from the nutritional intervention itself. In addition, the two-time randomization method may decrease the influence of the result of first randomization on the intension to exercise training.

In the current study the effect of a promising new intervention will be assessed to determine whether BCAA addition improve exercise capacity in patients with heart failure, who are generally complicated with sarcopenia. We hope this study, with its design and data collection, will present novel insights addressing the effective nutritional intervention in cardiac rehabilitation.

\section{Abbreviations}

AT: Anaerobic threshold; BCAA: Branched-chain amino acid;

CBC: Complete blood count; CHF: Chronic heart failure; Co-

I: Coinvestigator; CPX: Cardiopulmonary exercise test; EDC: Electronic

data capture; EF: Ejection fraction; HADS: Hospital anxiety and depression scale; ICH-GCP: International Conference on Harmonization of technical requirements for registration of pharmaceuticals for human use; IL-

6: Interleukin-6; PI: Principal investigator; SAEs: Serious adverse events; TNF: Tumor necrosis factor

\section{Acknowledgements \\ Not applicable.}

\section{Funding}

This work was supported by The Ministry of Education, Culture, Sports, Science and Technology of Japan through grant-in-aid 15 K19368 (to

Takata M) and 26,461,103 (to Amiya E)

\section{Availability of data and materials}

Not applicable.

\section{Authors' contributions}

MT and EA participated in the design of the study, conducted the trial and drafted the manuscript. YU participated in the design of the study and helped to draft the manuscript in particular statistics section. MW, YH, AN, TF, MT, GO, KH, NT, NT helped the conduction of this study. Data will be monitored by TM. IK supervise the study and revise the manuscript. All authors read and approved the final manuscript.

\section{Ethics approval and consent to participate}

The protocol was approved by the institutional review board of Tokyo University Hospital (P2015019-11X). Written informed consent is obtained from all participants prior to their participation in the study.

Consent for publication

Not applicable.

\section{Competing interests}

The authors declare that they have no competing interests.

\section{Publisher's Note}

Springer Nature remains neutral with regard to jurisdictional claims in published maps and institutional affiliations.

\section{Author details}

${ }^{1}$ Department of Cardiovascular Medicine, Graduate School of Medicine, The University of Tokyo, 7-3-1 Hongo, Bunkyo-ku, Tokyo 113-8655, Japan. ${ }^{2}$ Clinical Research Support Center, The University of Tokyo Hospital, Tokyo, Japan.

${ }^{3}$ Nursing Department, The University of Tokyo Hospital, Tokyo, Japan.

${ }^{4}$ Department of Rehabilitation, The University of Tokyo Hospital, Tokyo,

Japan. ${ }^{5}$ Suxac Inc., Tokyo, Japan.

Received: 7 June 2017 Accepted: 20 July 2017

Published online: 27 July 2017

\section{References}

1. Kokkinos PF, Choucair W, Graves P, Papademetriou V, Ellahham S. Chronic heart failure and exercise. Am Heart J. 2000;140(1):21-8. Review PubMed PMID:10874259

2. Cicoira M, Zanolla L, Franceschini L, Rossi A, Golia G, Zamboni M, Tosoni P, Zardini P. Skeletal muscle mass independently predicts peak oxygen consumption and ventilatory response during exercise in noncachectic patients with chronic heart failure. J Am Coll Cardiol. 2001;37(8):2080-5. PubMed PMID:11419891

3. Dos Santos MR, Saitoh M, Ebner N, Valentova M, Konishi M, Ishida J, Emami A, Springer J, Sandek A, Doehner W, Anker SD, von Haehling S. Sarcopenia and endothelial function in patients with chronic heart failure: results from the studies investigating comorbidities aggravating heart failure (SICA-HF). J Am Med Dir Assoc. 2017;18(3):240-5.

4. Fülster S, Tacke M, Sandek A, Ebner N, Tschöpe C, Doehner W, Anker SD, von Haehling S. Muscle wasting in patients with chronic heart failure: results from the studies investigating co-morbidities aggravating heart failure (SICA-HF). Eur Heart J. 2013;34(7):512-9. doi:10.1093/eurheartj/ehs381. Epub 2012 Nov 23.PubMed PMID: 23178647

5. Gitt AK, Wasserman K, Kilkowski C, Kleemann T, Kilkowski A, Bangert M, Schneider S, Schwarz A, Senges J. Exercise anaerobic threshold and 
ventilatory efficiency identify heart failure patients for high risk of early death. Circulation. 2002;106(24):3079-84. PubMed PMID: 12473555

6. Belardinelli R, Georgiou D, Cianci G, Purcaro A. Randomized, controlled trial of long-term moderate exercise training in chronic heart failure: effects on functional capacity, quality of life, and clinical outcome. Circulation. 1999;99(9):1173-1182. PubMed PMID: 10069785.

7. Sullivan MJ, Higginbotham MB, Cobb FR. Exercise training in patients with severe left ventricular dysfunction. Hemodynamic and metabolic effects. Circulation. 1988;78(3):506-15. PubMed PMID: 3409495

8. Coats AJ, Adamopoulos S, Radaelli A, McCance A, Meyer TE, Bernardi L, Solda PL, Davey P, Ormerod O, Forfar C, et al. Controlled trial of physical training in chronic heart failure. Exercise performance, hemodynamics, ventilation, and autonomic function. Circulation. 1992;85(6):2119-2131. PubMed PMID: 1591831

9. Hambrecht R, Fiehn E, Weigl C, Gielen S, Hamann C, Kaiser R, Yu J, Adams V, Niebauer J, Schuler G. Regular physical exercise corrects endothelial dysfunction and improves exercise capacity in patients with chronic heart failure. Circulation. 1998;98(24):2709-15. PubMed PMID: 9851957

10. O'Connor CM, Whellan DJ, Lee KL, Keteyian SJ, Cooper LS, Ellis SJ, Leifer ES, Kraus WE, Kitzman DW, Blumenthal JA, Rendall DS, Miller NH, Fleg JL, Schulman KA,McKelvie RS, Zannad F, Piña IL; HF-ACTION Investigators. Efficacy and safety of exercise training in patients with chronic heart failure: HF-ACTION randomized controlled trial. JAMA. 2009;301 (14):1439-1450. doi: 10.1001/jama.2009.454.PubMed PMID: 19351941; PubMed Central PMCID: PMC2916661.

11. Shimomura Y, Yamamoto Y, Bajotto G, Sato J, Murakami T, Shimomura N, Kobayashi H, Mawatari K. Nutraceutical effects of branched-chain amino acids on skeletal muscle. J Nutr. 2006;136(2):529S-32S.

12. Choudry HA, Pan M, Karinch AM, Souba WW. Branched-chain amino acidenriched nutritional support in surgical and cancer patients. J Nutr. 2006;136(1Suppl):314S-318S. Review. PubMed PMID: 16365105.

13. Howatson G, Hoad M, Goodall S, Tallent J, Bell PG, French DN. Exerciseinduced muscle damage is reduced in resistance-trained males by branched chain amino acids: a randomized, double-blind, placebo controlled study. J Int Soc Sports Nutr. 2012;9:20. doi:10.1186/15502783-9-20. eCollection 2012 PubMed PMID: 22569039; PubMed Central PMCID: PMC3395580

14. Tanada Y, Shioi T, Kato T, Kawamoto A, Okuda J, Kimura T. Branched-chain amino acids ameliorate heart failure with cardiac cachexia in rats. Life Sci. 2015;137:20-7. doi:10.1016/j.lfs.2015.06.021. Epub 2015 Jul 2 PubMed PMID: 26141987

15. Piepoli MF, Corrà U, Benzer W, Bjarnason-Wehrens B, Dendale P, Gaita D, McGee H, Mendes M, Niebauer J, Zwisler AD, Schmid JP. Cardiac Rehabilitation Section of the European Association of Cardiovascular Prevention and Rehabilitation. Secondary prevention through cardiac rehabilitation: from knowledge to implementation. A position paper from the Cardiac Rehabilitation Section of the European Association of Cardiovascular Prevention and Rehabilitation. Eur J Cardiovasc Prev Rehabil. 2010;17(1):1-17.

16. Dickinson JM, Volpi E, Rasmussen BB. Exercise and nutrition to target protein synthesis impairments in aging skeletal muscle. Exerc Sport Sci Rev. 2013:41(4):216-23.

\section{Submit your next manuscript to BioMed Central and we will help you at every step:}

- We accept pre-submission inquiries

- Our selector tool helps you to find the most relevant journal

- We provide round the clock customer support

- Convenient online submission

- Thorough peer review

- Inclusion in PubMed and all major indexing services

- Maximum visibility for your research

Submit your manuscript at www.biomedcentral.com/submit

C Biomed Central 